\title{
Epigenetic therapy with chidamide alone or combined with 5-azacitidine exerts antitumour effects on acute myeloid leukaemia cells in vitro
}

\author{
ZHENG LI $^{1,2^{*}}$, JIAN ZHANG $^{3 *}$, MIN ZHOU $^{4 *}$, JIN-LI LI $^{5}$, QIAO-CHENG QIU ${ }^{1,2}$, \\ JIAN-HONG FU ${ }^{1,2}$, SHENG-LI XUE ${ }^{1,2}$ and HUI-YING QIU ${ }^{1,2}$ \\ ${ }^{1}$ National Clinical Research Center for Hematologic Diseases, Jiangsu Institute of Hematology, \\ The First Affiliated Hospital of Soochow University; ${ }^{2}$ Institute of Blood and Marrow Transplantation, \\ Collaborative Innovation Center of Hematology, Soochow University; ${ }^{3}$ Department of Blood Transfusion, \\ The First Affiliated Hospital of Soochow University, Suzhou, Jiangsu 215006; ${ }^{4}$ Department of Hematology, \\ Changshu Affiliated Hospital of Soochow University, The First People's Hospital of Changshu, Changshu, \\ Jiangsu 215500; ${ }^{5}$ State Key Laboratory of Radiation Medicine and Protection, Oncology Radiotherapy Department, \\ The First Affiliated Hospital of Soochow University, Suzhou, Jiangsu 215006, P.R. China
}

Received September 14, 2021; Accepted January 4, 2022

DOI: $10.3892 /$ or.2022.8277

\begin{abstract}
Chidamide, a selective histone deacetylase inhibitor, has antitumour effects. 5-azacitidine (5-AZA), a hypomethylating agent, is effective in treating acute myeloid leukaemia (AML) and myelodysplastic syndrome. However, to the best of our knowledge, the effect of chidamide and 5-AZA on AML cell lines has not been fully investigated. In the present study, the antileukaemia activity of chidamide, alone and in combination with 5-AZA, was assessed on different subtypes of AML cell lines (M1-M5) and primary samples from several patients with AML in vitro. The results indicated that the proliferation of leukaemia cells was significantly and dose-dependently inhibited by chidamide and 5-AZA alone or in combination. The combination also had marked synergistic effects to induce apoptosis of AML cells. The apoptosis of leukaemia cells was induced via downregulation of BCL-2 and myeloid-cell leukemia 1 (MCL-1) levels. Of note, chidamide also degraded the MCL-1 protein in venetoclax-resistant U937 cells, in which the MCL-1 protein is upregulated. In addition,
\end{abstract}

Correspondence to: Professor Sheng-Li Xue or Professor Hui-Ying Qiu, National Clinical Research Center for Hematologic Diseases, Jiangsu Institute of Hematology, The First Affiliated Hospital of Soochow University, 188 Shizi Street, Suzhou, Jiangsu 215006, P.R. China

E-mail: slxue@suda.edu.cn

E-mail: qiuhuiying@aliyun.com

*Contributed equally

Key words: chidamide, 5-azacitidine, proliferation, differentiation, apoptosis, acute myeloid leukaemia chidamide was able to induce myeloid differentiation (with CD11b upregulation) of AML cell lines or monocytic/dendritic differentiation (with CD86 upregulation) of primary cultured cells from several patients with AML. Chidamide was also able to promote the differentiation of the venetoclax-resistant U937 cell line by upregulating CD11b expression. In conclusion, chidamide alone or combined with 5-AZA may be an effective therapy for AML.

\section{Introduction}

Numerous studies have indicated that cancer may be caused both by mutations in DNA and by two specific types of ubiquitous epigenetic modifications: DNA methylation and histone acetylation $(1,2)$. These two epigenetic modifications may regulate gene expression, remodel chromatin, alter cell phenotypes and promote cancer development $(3,4)$. In addition, methylation and acetylation modifications may regulate cellular pathways that are involved in cell differentiation, proliferation and apoptosis, which are attributed to the genesis of leukaemia (5).

DNA methylation has been widely studied in DNA epigenetic modification. In acute myeloid leukaemia (AML), abnormal DNA methylation may silence the expression of tumour suppressor genes by binding to the upstream promoter region (6). Changes in DNA methylation are prevalent in myelodysplastic syndrome (MDS) and secondary AML and they appear to have an important role in the transformation of MDS to AML (7). Hence, hypomethylating agents (HMAs) are important in the treatment of higher-risk patients with MDS and AML, particularly in those patients who are ineligible for haematopoietic stem cell transplantation $(8,9)$. 5-azacitidine (5-AZA), which is a cytidine analogue prodrug, is an HMA for the treatment of higher-risk MDS and/or AML. 
Histone deacetylases (HDACs) are able to alter gene expression and chromatin modification by inducing the deacetylation of histones and other processes (10). In addition, the conversion of DNA-histone complexes from an open state to a compact configuration is closely related to gene transcription silencing (11). In addition to histone deacetylation-induced gene silencing, DNA methylation is also engaged in histone methylation, which is another important epigenetic modification and may regulate proliferation, ageing, tumourigenesis and other biological processes (12). Chidamide is an original new drug in China and a novel selective HDAC inhibitor (HDACI). It is able to selectively inhibit HDAC-1, -2, -3 and -10 , particularly -2 and -3 . Chidamide has been researched in numerous clinical trials in the USA and China and has been ratified to treat cutaneous T-cell lymphoma and peripheral T-cell lymphoma in China (13). Chidamide is highly toxic to leukaemia cells in a concentration-dependent manner, and less toxic, more tolerated and more stable than other drugs (14). An increasing number of studies have confirmed its antileukaemia effect. Both DNA methylation and histone acetylation are reversible to a certain extent (2), which is worth considering for the design of therapeutic strategies. This mechanism suggests that the combination of HDACI and HMA may be effective against leukaemia. Therefore, HDACI and HMA have become novel strategies for leukaemic and epigenetic treatments in AML (15).

In the present study, the antileukaemia effects of chidamide alone and in combination with 5-AZA on AML cells were assessed. The results suggested that chidamide and its combination with 5-AZA had a strong antileukaemic effect by inhibiting cell proliferation and by inducing apoptosis of AML cells. These results revealed that chidamide is a potential drug for leukaemia treatment, particularly in combination with 5-AZA.

\section{Materials and methods}

Cell lines, primary AML samples and reagents. The AML cell lines KG1a, Kasumi-1, NB4, OCI-AML3, U937, MV4-11 and SKM1 were obtained from the Cell Biology Research Institute (Chinese Academy of Sciences) (Table SI). U937 was validated by short tandem repeat analysis. The cell lines were cultured in RPMI 1640 containing $10 \%$ foetal bovine serum (FBS) (Gibco; Thermo Fisher Scientific, Inc.), $100 \mathrm{U} / \mathrm{ml}$ penicillin and $100 \mu \mathrm{g} / \mathrm{ml}$ streptomycin in an incubator at $37^{\circ} \mathrm{C}$ in a humidified atmosphere consisting of $95 \%$ air and $5 \% \mathrm{CO}_{2}$.

Primary AML cells were isolated from peripheral blood (PB) or bone marrow (BM) containing $>50 \%$ blasts. These specimens were cultured in IMDM (Gibco; Thermo Fisher Scientific, Inc.) containing 10\% FBS, $50 \mathrm{ng} / \mathrm{ml}$ recombinant human (rh) stem cell factor, $100 \mathrm{ng} / \mathrm{ml} \mathrm{fms-related} \mathrm{receptor}$ tyrosine kinase 3 (FLT3) ligand, $25 \mathrm{ng} / \mathrm{ml} \mathrm{rhIL}-3$ and $10 \mathrm{ng} / \mathrm{ml}$ rhIL-6 (PeproTech, Inc.). The present study was approved by the Research Ethics Committee of The First Affiliated Hospital of Soochow University (Suzhou, China) and written informed consent was provided by all subjects.

Chidamide (Chipscreen Biosciences Ltd.) was dissolved in DMSO (Thermo Fisher Scientific, Inc.) to a concentration of $20 \mathrm{mM}$ to prepare a stock solution. The stock solution was stored at $-80^{\circ} \mathrm{C}$. 5-AZA (MilliporeSigma) and Venetoclax
(MCE MedChemExpress) was respectively dissolved in DMSO to a $100 \mathrm{mM}$ concentration and kept at $-80^{\circ} \mathrm{C}$. The stock solutions were diluted to working concentrations in subsequent experiments with growth media.

Mononuclear cell separation. BM or PB samples were collected from 5 patients (P1: Male, 57 years; P2: Male, 81 years; P3: Female, 55 years; P4: Female, 75 years; P5: Male, 56 years) who had been diagnosed with AML (non-acute promyelocytic leukaemia) at the First Affiliated Hospital of Soochow University between January 2020 to May 2021 (Table SII). Mononuclear cells were separated by using Ficoll solution (TBD Science) according to the manufacturer's protocol (16).

Cell viability assay. The cytotoxic effects of chidamide and 5-AZA alone (or the combination of the two drugs) on the AML cell lines were determined via the use of the Cell Counting Kit-8 (CCK-8) assay (Dojindo Laboratories, Inc.). Cell lines were seeded at a density of $1-3 \times 10^{4} / \mathrm{ml}$ in 96 -well flat-bottomed microtiter plates at $100 \mu \mathrm{l} /$ well and exposed to chidamide $(0-20 \mu \mathrm{M})$ or 5-AZA $(0-40 \mu \mathrm{M})$ at varying concentrations (either alone or in combination) for $72 \mathrm{~h}$, after which they were subjected to a standard CCK- 8 assay. The plate was read at a wavelength of $490 \mathrm{~nm}$ using a microplate reader (Bio-Rad Laboratories, Inc.).

Cell cycle analysis. The cells were treated with chidamide $(1 \mu \mathrm{M})$ for $48 \mathrm{~h}$. Subsequently, the cells were collected, washed with PBS and then fixed overnight in $75 \%$ ice-cold ethanol at $4^{\circ} \mathrm{C}$. The fixed cells were then harvested, stained with propidium iodide (PI)/RNase A (BD Pharmingen; BD Biosciences) and incubated in the dark at room temperature for $30 \mathrm{~min}$ after being washed with PBS. The DNA content was analyzed by flow cytometry (FCM) with an LSR2 instrument (BD Biosciences). FlowJo 7.6 software (Tree Star, Inc.) was used for data analysis.

Total RNA isolation and reverse transcription-quantitative $(R T-q) P C R$. The cells were treated with chidamide $(1 \mu \mathrm{M})$ for $72 \mathrm{~h}$ and total cellular RNA was extracted from the cells using Direct-zol ${ }^{\mathrm{TM}}$ RNA MircoPrep (ZYMO Research Corp.) according to the manufacturer's protocols. RNA was eluted with RNase-free water, quantified at an absorbance at 260/280 $\mathrm{nm}$ and subjected to RT. Total mRNA was reverse transcribed into cDNA using a SuperScript ${ }^{\mathrm{TM}}$ IV One-Step RT-PCR System (cat. no. 12594025; Thermo Fisher Scientific, Inc.). Real-time qPCR was performed using TaqMan master mix (Thermo Fisher Scientific, Inc.) with $0.2 \mathrm{mM}$ TaqMan probe (Thermo Fisher Scientific, Inc.). The real-time qPCR conditions were as follows: 1 cycle at $50^{\circ} \mathrm{C}$ for $2 \mathrm{~min}, 1$ cycle at $95^{\circ} \mathrm{C}$ for $20 \mathrm{sec}$ and 40 cycles at $95^{\circ} \mathrm{C}$ for $1 \mathrm{sec}$ and $60^{\circ} \mathrm{C}$ for $20 \mathrm{sec}$. Signals were detected with a QuantStudio 7 Flex Real-Time PCR system (Thermo Fisher Scientific, Inc.). Relative expression levels were determined by normalizing to GAPDH levels. GAPDH-qPCR Taqman probe (cat. no. Hs02786624_g14453320; Thermo Fisher Scientific, Inc.) and early growth response 1 (EGR1)-qPCR Taqman probe (cat. no. Hs00152928_m14331182; Thermo Fisher Scientific, Inc.) were used. The results were analyzed using the 
$2^{-\Delta \Delta \mathrm{Cq}}$ method, in which $\Delta \mathrm{Cq}=\mathrm{Cq}$ (target gene) $\mathrm{Cq}$ (internal reference) and $\Delta \Delta \mathrm{Cq}=\Delta \mathrm{Cq}$ (sample) $-\Delta \mathrm{Cq}$ (control) (17). Each sample was measured in triplicate.

Cell apoptosis assay. The effects of chidamide alone or combined with 5-AZA on apoptosis of AML cell lines were analysed by using FCM analysis. In brief, cells were harvested, washed with cold PBS and resuspended in $400 \mu \mathrm{l}$ Annexin Binding Buffer. Subsequently, $1 \mu$ l allophycocyanin (APC)-conjugated Annexin V and $5 \mu \mathrm{l}$ PI (BD Pharmingen; BD Biosciences) were added to each sample. Stained samples were analysed by using FCM with an LSR2 instrument (BD Biosciences).

AML cell differentiation analysis. AML cell lines and AML patient samples were treated with chidamide for three days prior to analysis. Phycoerythrin/cyanin 7-conjugated mouse anti-human CD11b monoclonal antibody (clone ICRF44; cat. no. 301322; BioLegend, Inc.) and APC-conjugated mouse anti-human CD86 monoclonal antibody (clone IT2.2; cat. no. 305412; BioLegend, Inc.) were used for staining at a 1:200 dilution $\left(30 \mathrm{~min}\right.$ at $\left.4^{\circ} \mathrm{C}\right)$. Stained samples were analysed by using FCM with an LSR2 instrument (BD Biosciences). The results were then analysed with the use of FlowJo 7.6 software (TreeStar, Inc.).

Western blot analysis. Cultured cells were harvested, washed with PBS and then lysed in RIPA buffer. The protein lysates were clarified via centrifugation at $12,000 \mathrm{x}$ f for $30 \mathrm{~min}$ at $4^{\circ} \mathrm{C}$ and the supernatant was collected. The protein concentration was measured by using a BCA Protein Assay Kit (Beyotime Institute of Biotechnology). Equal amounts (30 $\mu$ g per lane) of protein were separated by using $12 \%$ SDS/PAGE, after which they were electrotransferred onto a PVDF membrane (MilliporeSigma). The membranes were blocked with 5\% skimmed milk (BD Biosciences) and incubated with primary antibodies at $4{ }^{\circ} \mathrm{C}$ overnight. The primary antibodies were as follows: BCL-2 (cat. no. sc-7382; dilution, 1:500; Santa Cruz Biotechnology, Inc.), BAX (cat. no. sc-7480; dilution, 1:500; Santa Cruz Biotechnology, Inc.), BCL-XL (cat. no. sc-8392; dilution, 1:500; Santa Cruz Biotechnology, Inc.) and MCL-1 (cat. no. sc-12756; dilution, 1:500; Santa Cruz Biotechnology, Inc.). GAPDH (cat. no. 97166; dilution, 1:1,000; Cell Signaling Technology, Inc.) was used as a loading control. The membrane was then incubated with secondary antibodies (cat. no. A0216; dilution, 1:1,000; Beyotime Institute of Biotechnology) for $1 \mathrm{~h}$ at room temperature, and visualized with the use of an enhanced chemiluminescent western blotting detection reagent (cat. no. RPN2209; Cytiva) via an Gel Doc ${ }^{\mathrm{TM}}$ EZ imaging system (Bio-Rad Laboratories, Inc.).

Statistical analysis. All of the experiments were performed in triplicate wells as three independent replicates. All of the data are presented as the mean \pm standard deviation. One-way ANOVA was used to compare multiple independent groups by GraphPad Prism 5 (GraphPad Software, Inc.). Further statistical comparisons were performed by using Bonferroni's and Dunnett's test and the corresponding bar charts or linear graphs were drawn by using GraphPad Prism 5 (GraphPad Software, Inc.). $\mathrm{P}<0.05$ was considered to indicate a statistically significant difference. The half-maximal inhibitory concentration $\left(\mathrm{IC}_{50}\right)$ values were calculated with GraphPad. The combination index (CI) value was calculated from the fraction-affected value of each combination (according to the Chou-Talalay method) by using CompuSyn software 1.0.1 (ComboSyn, Inc.).

\section{Results}

Chidamide and 5-AZA synergistically inhibit the proliferation of AML cell lines and primary cultured AML cells. To determine the effect of chidamide and 5-AZA on proliferation, the $\mathrm{IC}_{50}$ values for chidamide and 5-AZA alone were first determined at different concentrations when applied to the AML cell lines M1-M5 for $72 \mathrm{~h}$. The M1-M5 cell lines represent different AML subtypes in accordance with the French-American-British (FAB) classification (Table SI). Chidamide and 5-AZA caused growth inhibition of the M1-M5 cell lines in a dose-dependent manner (Fig. 1A and B). Chidamide markedly inhibited AML cell proliferation at low concentrations (except for KG1a). By contrast, KG1a and Kasumi-1 cells were insensitive to 5-AZA compared with the other cell lines.

Next, the effect of the chidamide and 5-AZA combination on cell viability was evaluated. Cell lines were treated with different concentrations, in accordance with the $\mathrm{IC}_{50}$ of each drug. Chidamide plus 5-AZA inhibited AML cell proliferation in a dose-dependent manner and the combination had a stronger inhibitory effect than either drug alone (Fig. 2A-F). In addition, chidamide and 5-AZA (alone or in combination) had marked cytotoxic effects on the proliferation of primary cultured AML cells from patients and the inhibitory effect was dose-dependent (Fig. 2G and H). The calculation of the CI value suggested that chidamide combined with 5-AZA also had a clear synergistic effect at their suitable concentrations (Fig. 2I). Furthermore, the results of the cell cycle analysis indicated that chidamide was able to cause G1 phase arrest in the MV4-11 cell line (Fig. S1).

Apoptosis is significantly induced by chidamide plus 5-AZA in AML cell lines. An FCM analysis was performed to determine the ability of the drugs to induce apoptosis in AML cells. It was observed that the percentage of Annexin V-positive cells significantly increased in the M1-M5 cell line at $72 \mathrm{~h}$ after the use of chidamide or 5-AZA treatment as well as with the combination treatment (Fig. 3A and B). Of note, treatment with chidamide plus 5-AZA induced significantly higher rates of apoptosis than single treatments with either drug (Fig. 3C). The results also suggested that chidamide plus 5-AZA not only increased the early apoptotic population (Annexin $\mathrm{V}^{+} / \mathrm{PI}^{-}$) in KG1a, Kasumi-1, U937 and MV4-11 cells [as well as the late apoptotic population (Annexin $\mathrm{V}^{+} / \mathrm{PI}^{+}$) in NB4 cells], but also caused increases in both early and late apoptotic populations in OCI-AML3 cells.

Chidamide induces the differentiation of AML cell lines and primary AML samples. To investigate the effect of chidamide on AML cell differentiation, the ability of the drug to upregulate the differentiation markers CD11b (integrin subunit $\alpha \mathrm{M}$ ) and CD86 was assessed in AML cells. CD11b is a myeloid differentiation marker and CD86 is a monocytic/dendritic 

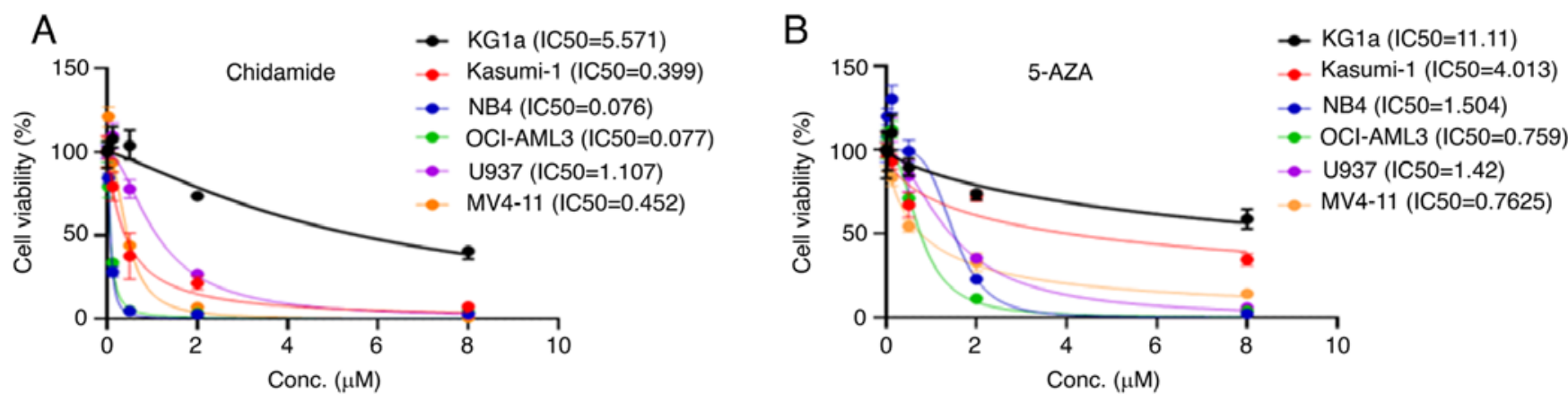

Figure 1. Chidamide and 5-AZA alone inhibit cell proliferation in different AML cell lines. All of the AML cell lines were exposed to (A) chidamide or (B) 5-AZA for $72 \mathrm{~h}$ and cell viability was subsequently determined by using a Cell Counting Kit-8 assay. 5-AZA, 5-azacitidine; AML, acute myeloid leukaemia; Conc., concentration.

differentiation marker. Analysis of The Cancer Genome Atlas (TCGA) (https://portal.gdc.cancer.gov/) and TAGERT (https://ocg.cancer.gov/programs/target) databases indicated that from the M1 to M5 subtypes of AML, the expression levels of CD11b and CD86 gradually increased at different stages of cell differentiation and maturation (Fig. 4A). Chidamide induced the expression of CD11b in Kasumi-1, NB4, OCI-AML3, U937 and MV4-11 cells (Fig. 4B). Chidamide also upregulated the expression of CD86 (4-fold increase) in primary AML cells but the effect on CD11b (2-fold increase) expression was only slight (Fig. 4C). To investigate the mechanism of differentiation, additional experiments were performed, which indicated that chidamide upregulates the expression of EGR-1 in U937 cells (Fig. S2).

Chidamide and 5-AZA downregulate the antiapoptotic proteins BCL-2 and MCL-1 in AML cell lines. BCL-2 family proteins are critical regulators of apoptotic pathways. To understand the molecular mechanism underlying chidamideand 5-AZA-induced apoptosis, western blot assays were performed, which indicated that AML cell lines exhibited decreased levels of antiapoptotic proteins (BCL-2 and MCL-1) in response to the single drug treatments after $72 \mathrm{~h}$ (Fig. 5A). Chidamide also downregulated the expression of BCL-XL and upregulated BAX in MV4-11 and Kasumi-1 (Fig. S3).

Chidamide downregulates BCL-2 and induces the differentiation of the venetoclax-resistant U937 cell line. Venetoclax markedly inhibited the proliferation of different AML cell lines (M1-M5) at low concentrations (except for U937; $\mathrm{IC}_{50}=6 \mu \mathrm{M}$ ) (Fig. 5B); thus, the U937 cell line was selected for further analysis. Next, a venetoclax-resistant U937 cell line (R-VEN) was established by gradually increasing the concentration of venetoclax to $12 \mu \mathrm{M}$ in vitro. Western blot analysis suggested that the expression of MCL-1 protein increased in R-VEN; however, exposure to chidamide led to a modest reduction in MCL-1 expression in R-VEN cells (Fig. 5C). Furthermore, chidamide upregulated the expression of CD11b in the R-VEN cell line (Fig. 5D).

\section{Discussion}

From the 1970s until 2017, the progress in the frontline treatment of AML was limited. In other words, since the establishment of ' $7+3$ ' chemotherapy (cytarabine and anthracycline) in the mid-1970s, the development of agents for patients with AML has proven to be a daunting challenge (18). With improvements in supportive care, long-term survival has improved in younger patients with AML, with a 5-year overall survival (OS) in the range of $40-50 \%$. However, among patients above the age of 60 years who make up the bulk of AML cases, the long-term outlook is dismal, with a 5-year OS of 10-20\% (19). Therefore, there remains to be a clear requirement for newer therapies and a more individualized approach for the treatment of AML.

Genes that may be silenced by abnormal methylation or acetylation modifications are promising targets for cancer therapies, particularly in AML (20). In clinical practice, using HDACIs and HMAs in combination or with other antitumour drugs may be an effective antileukaemia strategy. To explore this, clinical trials of HMA combined with HDACI or other drugs for AML have been performed (21-23). HDACI plus HMA has already demonstrated promising effects in the clinical treatment of AML (11,24,25).

In the present study, different types of AML cell lines were used as models, and chidamide and 5-AZA alone or in combination inhibited the proliferation of the M1-M5 AML cell lines in a dose-dependent manner. Different cell lines represent different subtypes of the AML FAB classification. MV4-11 represent patients harbouring the FLT3-internal tandem duplication mutation, which has the highest mutation frequency in AML. When compared with other AML cell lines, both drugs had a weak effect on the growth inhibition of KG1A cells and the $\mathrm{IC}_{50}$ values of chidamide and 5-AZA were 5.57 and $11.11 \mu \mathrm{M}$, respectively. The reason may be the slow growth rate of the cell itself; therefore, it is not sensitive to chemotherapy drugs. However, it remains elusive why Kasumi-1 was insensitive to 5-AZA compared with other cell lines; this may possibly be due to special cytogenetics (chromosome and gene mutations). When the two drugs were combined, the inhibitory effect on proliferation was more potent than that of a single drug and the significant synergistic effect was confirmed by the CI value at certain suitable concentrations. It was also observed that chidamide and 5-AZA alone or in combination markedly inhibited the proliferation of AML cells from patient samples; however, the time-point of the test was important, as it was observed that $72 \mathrm{~h}$ was too toxic to cells and the synergistic effect was not obvious, and the incubation time was thus decreased to $24 \mathrm{~h}$, at which the synergistic effect was more 
A

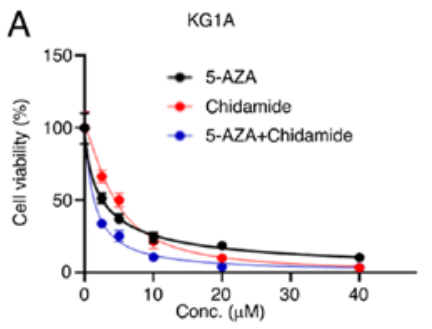

$\begin{array}{lllllll}\text { 5.AZA } & 0 & 2.5 & 5 & 10 & 20 & 40\end{array}$

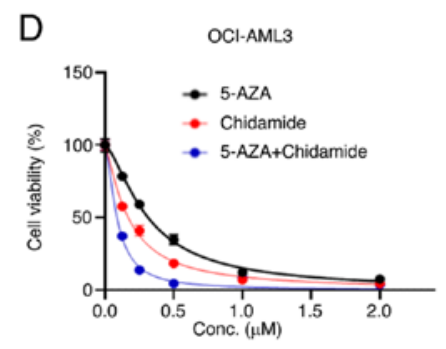

$\begin{array}{lllllll}\text { 5.AZA } & 0 & 0.125 & 0.25 & 10.5 & 1 & 2\end{array}$ $\begin{array}{llllll}\text { Chidamide } 0 & 0.025 & 0.05 & 0.1 & 0.2 & 0.4\end{array}$
B

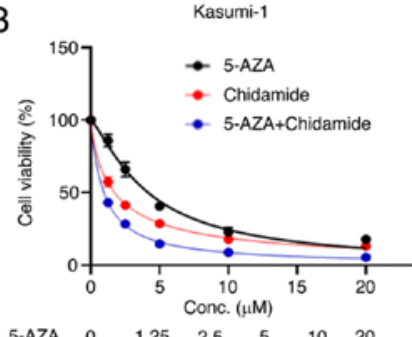

$\begin{array}{rrrrrrr}\text { 5-AZA } & 0 & 1.25 & 2.5 & 5 & 10 & 20\end{array}$

E

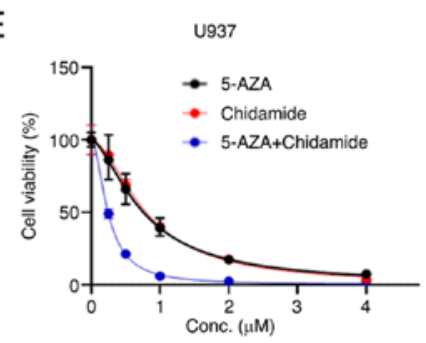

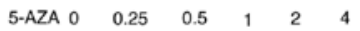

C

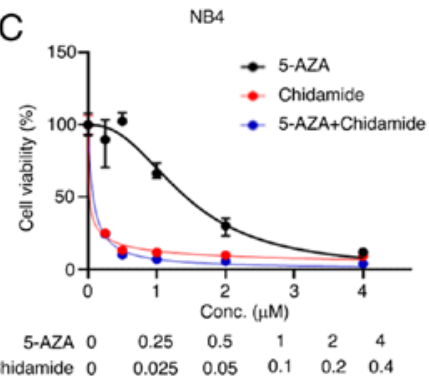

$\mathrm{F}$

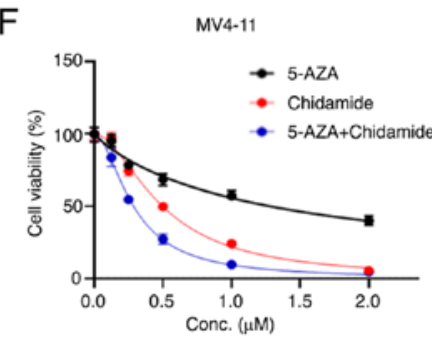

$\begin{array}{lllllll}\text { 5-AZA } & 0 & 0.125 & 0.25 & 0.5 & 1 & 2\end{array}$ $\begin{array}{lllllll}\text { Chidamide } & 0 & 0.125 & 0.25 & 0.5 & 1 & 2\end{array}$

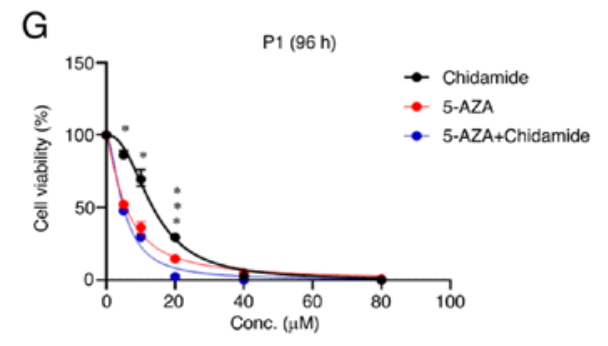

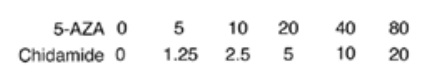
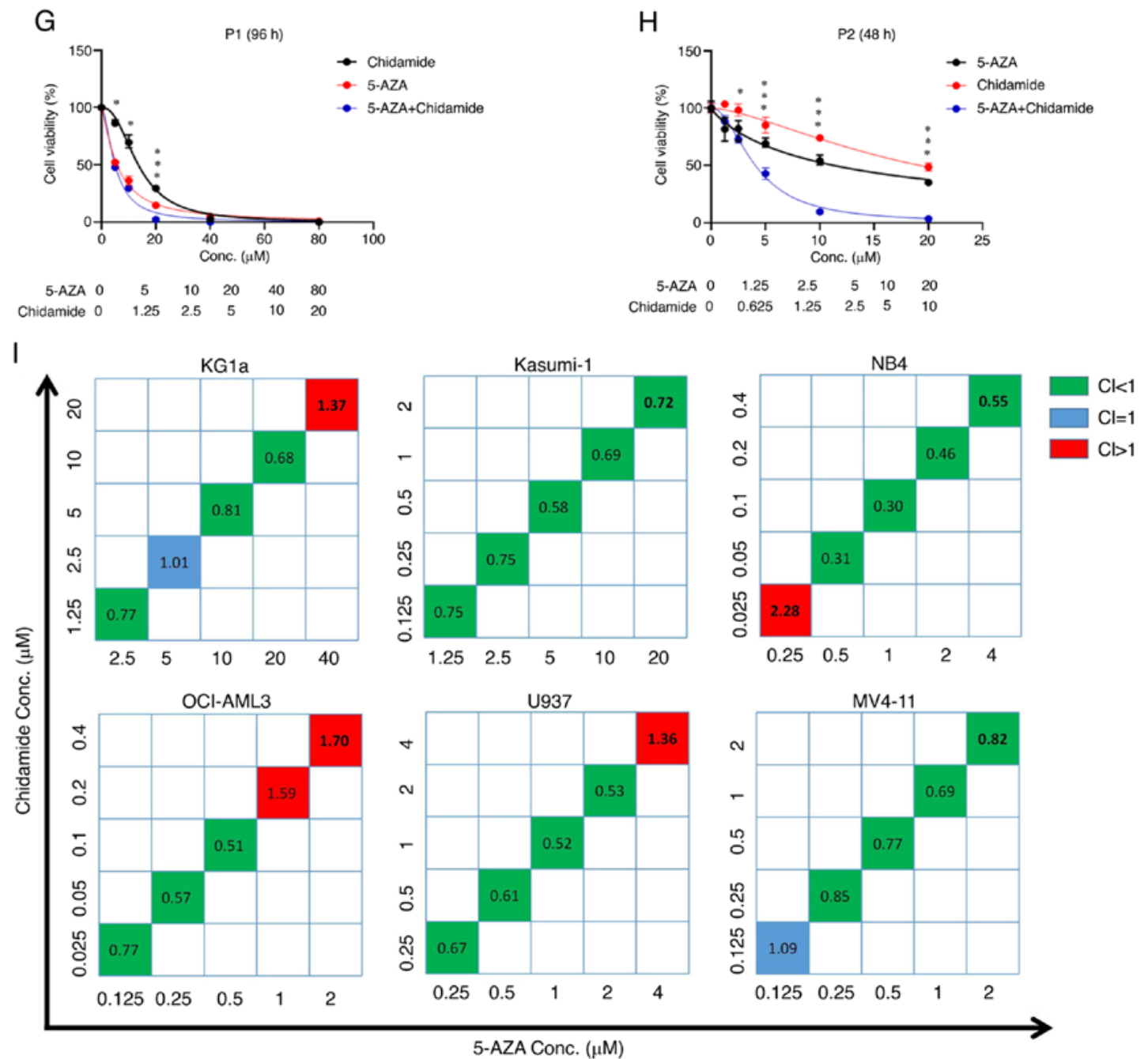

5-AZA Conc. $(\mu \mathrm{M})$

Figure 2. Chidamide, 5-AZA, and their combination inhibit cell proliferation in AML cells. (A) KG1a, (B) Kasumi-1, (C) NB4, (D) OCI-AML3, (E) U937 and (F) MV4-11 cell lines were exposed to chidamide, 5-AZA and their combination for $72 \mathrm{~h}$ and the cell viability was subsequently determined by using a Cell Counting Kit-8 assay. The curves represent the dose-dependent effects of chidamide and 5-AZA on cell proliferation. (G and $\mathrm{H}$ ) The two drugs in combination (5-AZA+Chidamide) exhibited a significant proliferation inhibition effect compared with each single drug (5-AZA and Chidamide) in AML primary samples of (G) P1 and (H) P2. ${ }^{*} \mathrm{P}<0.05,{ }^{* * *} \mathrm{P}<0.001$. (I) CI values for the two drugs in the KG1a, Kasumi-1, NB4, OCI-AML3, U937 and MV4-11 cell lines. The horizontal axis represents different concentrations of 5-AZA and the vertical axis represents different concentrations of chidamide. The CI value reflects the degree of drug interaction: $\mathrm{Cl}<1, \mathrm{CI}=1$ or $\mathrm{CI}>1$ indicated synergistic, additive or antagonistic effects, respectively. Different colours represent different $\mathrm{CI}$ values. P1, patient 1; 5-AZA, 5-azacitidine; AML, acute myeloid leukaemia; Conc., concentration; CI, combination index. 
A
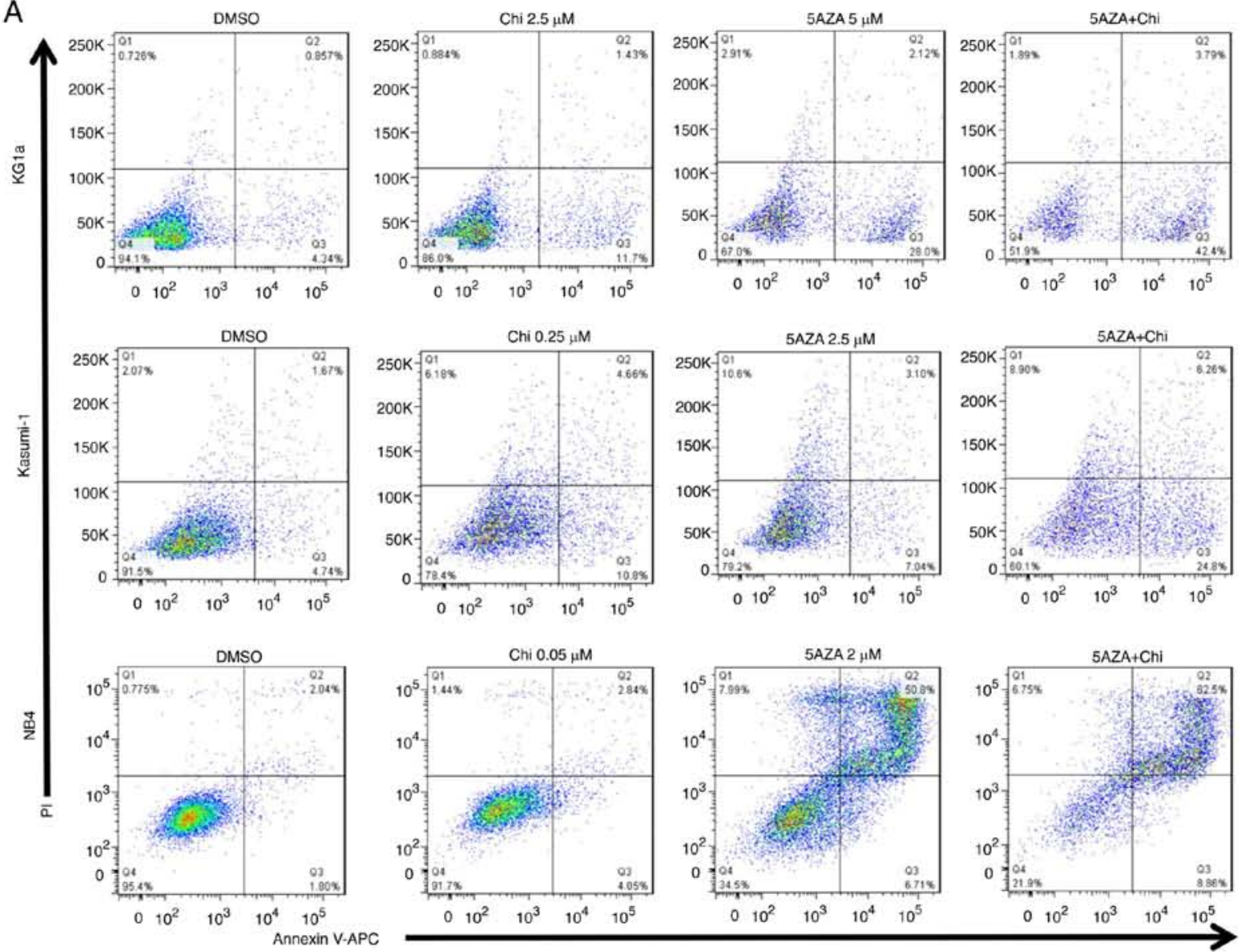

B
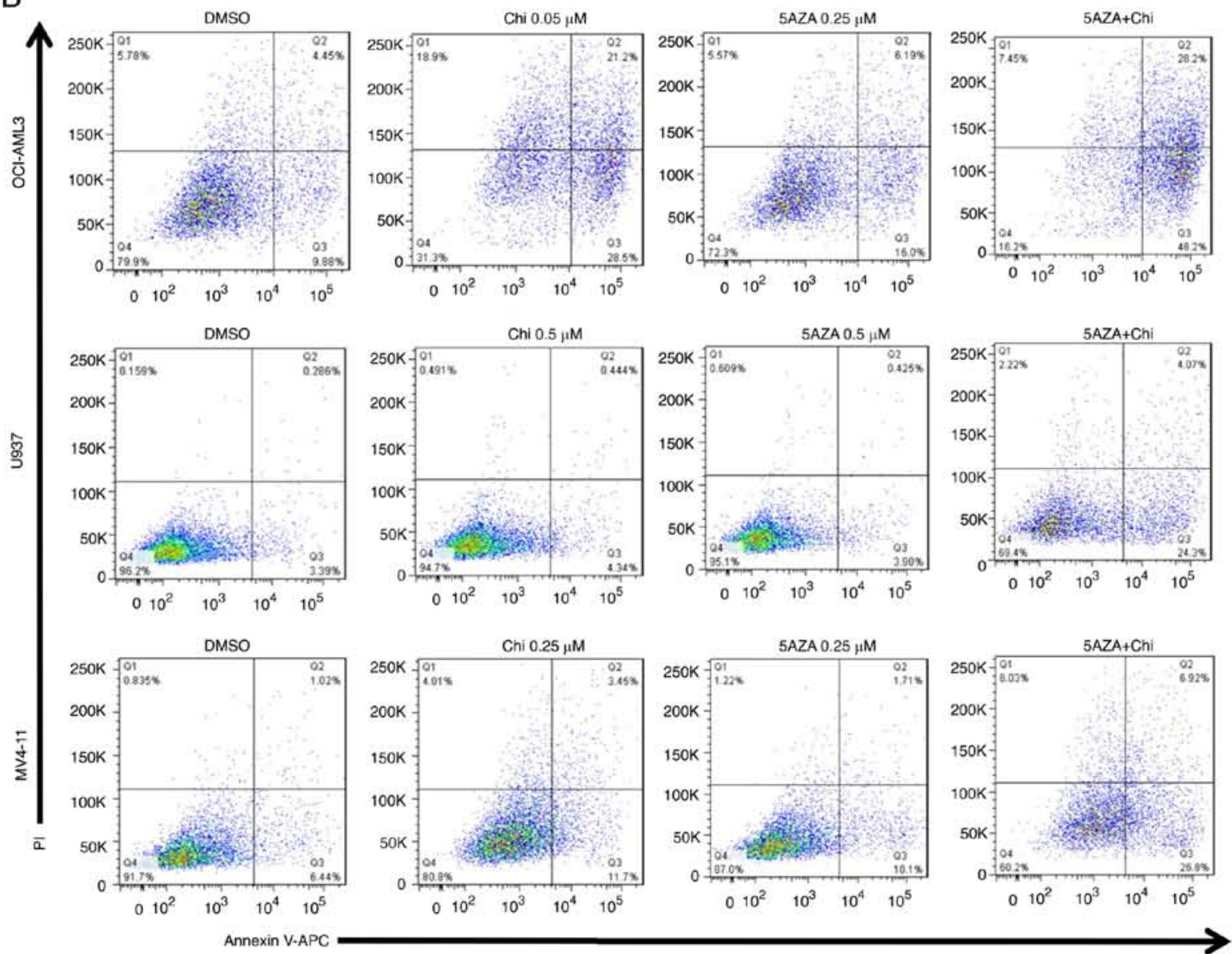

Figure 3. Continued. 

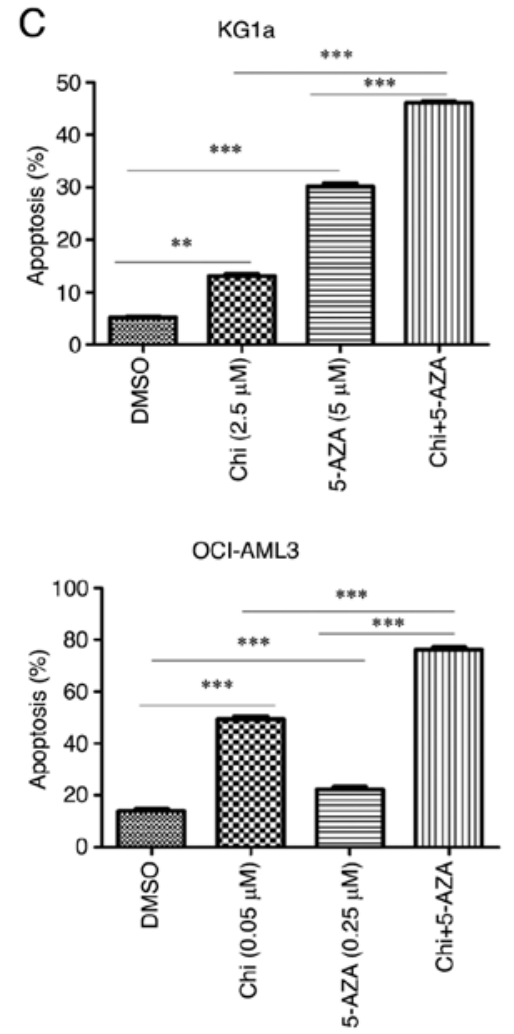
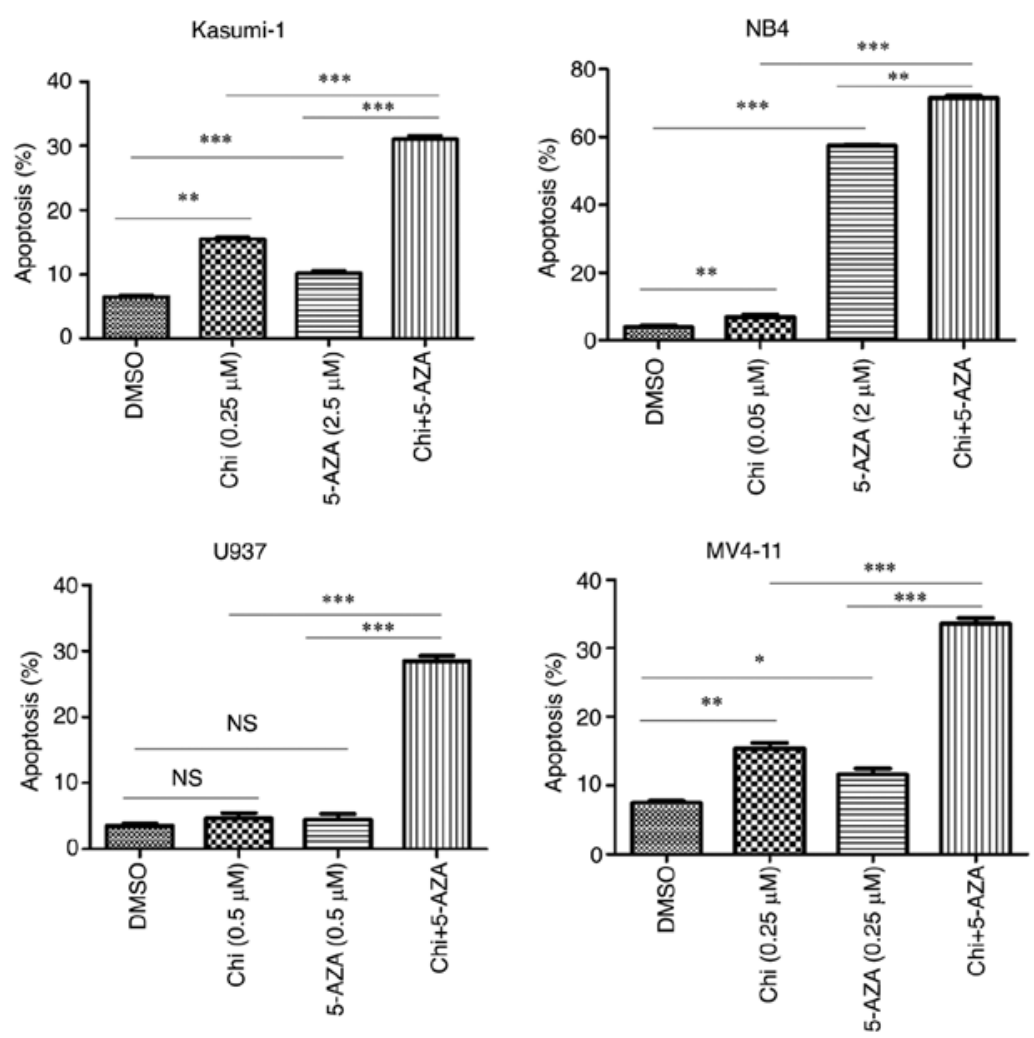

Figure 3. Chidamide induces apoptosis in AML cells and cooperatively induces apoptosis with 5-AZA. (A and B) Apoptosis was determined using Annexin-V/PI staining after AML cell lines were treated with chidamide alone and combined with 5-AZA at the indicated concentrations for 72 h. (A) KG1a, Kasumi-1 and NB4; (B) OCI-AML3, U937 and MV4-11. (C) The total percentage of apoptotic cells (Annexin-V \& PI double-positive) was measured. Values are expressed as the mean \pm standard deviation of three separate experiments. ${ }^{*} \mathrm{P}<0.05,{ }^{* *} \mathrm{P}<0.01$ and ${ }^{* * *} \mathrm{P}<0.001$. NS, no significance; 5-AZA, 5-azacitidine; Chi, chidamide; APC, allophycocyanin; Q, quadrant; AML, acute myeloid leukaemia.

obvious. In the subsequent experiment, the minimum value of the CI was not selected as the combination concentration, but the intermediate concentration combination was chosen for apoptosis in order to obtain a significant difference in the results. When measuring differentiation, a concentration close to the $\mathrm{IC}_{50}$ was selected, as changes in the expression rate of $\mathrm{CD} 11 \mathrm{~b}$ were most obvious with this concentration. Meanwhile, the chidamide-induced cell death did not affect the results of the differentiation assay.

Numerous studies have confirmed that HDACI induces cell cycle arrest in G0/G1 phase in vitro $(13,26,27)$. The experimental results of the present study suggested that chidamide is able to cause G1 phase arrest. HDACI and HMA are able to overcome drug resistance by reactivating silenced tumour suppressor genes and inducing cell cycle arrest (28). When chidamide was combined with 5-AZA, chidamide potently and synergistically enhanced the cytotoxicity in AML cell lines. This synergistic cytotoxicity was obvious, as the two agents were able to cooperatively induce cell apoptosis. The present study indicated that the single drug-induced inhibition was incomplete; however, when chidamide plus 5-AZA was added, the inhibition was significantly improved. Different cell lines had different apoptosis types; specifically, certain cell lines exhibited early apoptosis, while others exhibited late apoptosis, and certain cells exhibited both types of apoptosis.

The FAB classification system is based on morphology for defining specific immunotypes (29). From M1 to M5, the maturity of cells increased and the expression levels of CD11b and CD86 also tended to increase, which was also confirmed by the results from the TCGA and TARGET databases. CD11b is considered a myeloid differentiation marker of leukaemia cells (30). For instance, the expression of CD11b may be significantly upregulated when AML-M3 cells are treated with ATRA and a similar change may also be observed in U937 cells treated with Brequinar (31). CD86 is considered a monocytic/dendritic differentiation marker (32). The present results indicated that the expression of CD11b on Kasumi-1, NB4, OCI-AML3, U937 and MV4-11 cells was upregulated (except for KGla cells) after treatment with chidamide. Perhaps KG1a has different biological characteristics from other AML cell lines, thus making them more primitive and more difficult to differentiate, based on drug induction. Further research on the combined effect of chidamide and 5-AZA on the differentiation of AML cells requires to be performed. EGR-1 is a differentiation-related transcription factor. The results of the present study indicated that chidamide was able to upregulate the expression of EGR-1 in U937. However, when primary cultured cells from patients with AML were treated with chidamide in vitro, it was observed that both CD11b and CD86 were upregulated, thus indicating that the cells further differentiated into monocytic/dendritic cells. As most patients are diagnosed with M4 or M5, they are more likely to exhibit monocytic/dendritic differentiation. It was speculated that this may be related to the subtypes of AML patients, which requires further exploration. 


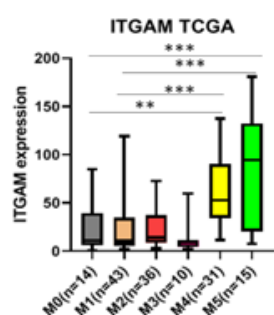

CD86 TCGA

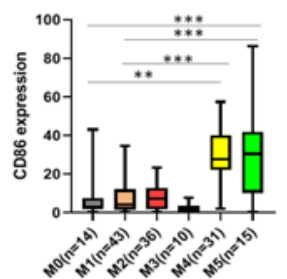

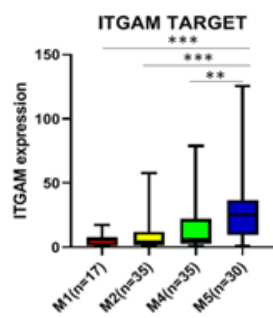

CD86 TARGET

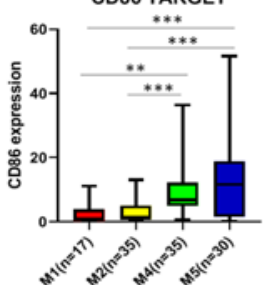

B

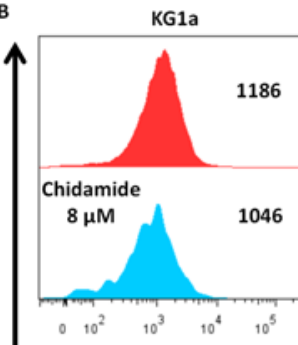

OCI/AML3

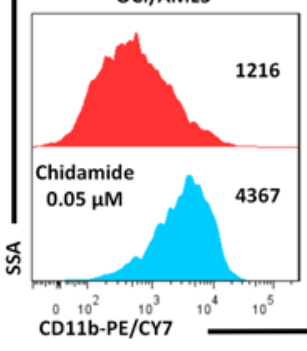

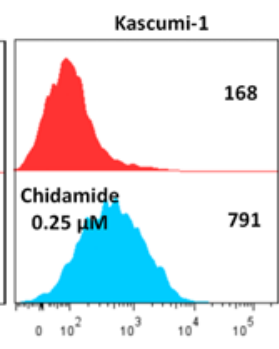

U937

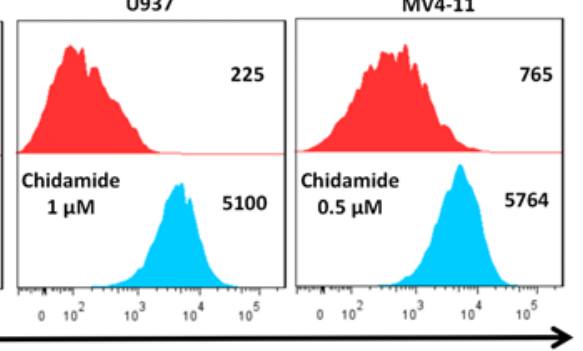

C

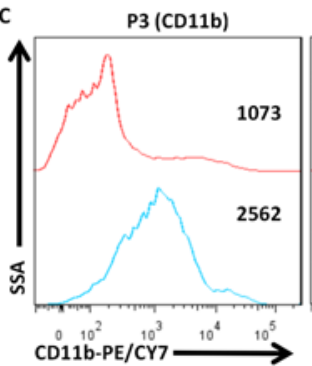

P3 (CD86) P4 (CD86) P5 (CD86)

- DMsO

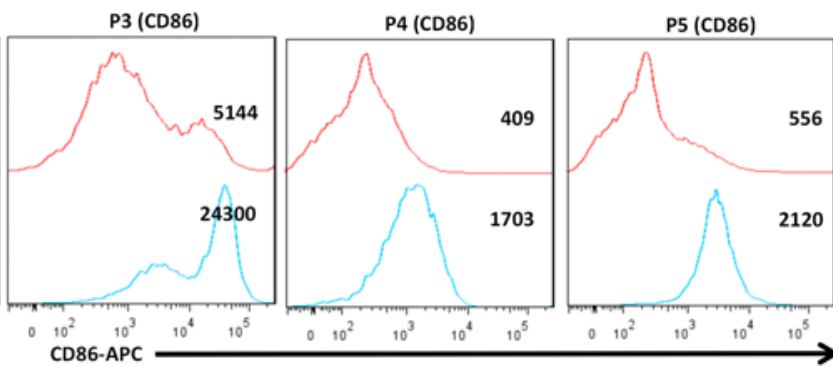

Figure 4. Differentiation induction activity of chidamide in AML cell lines and primary AML samples. (A) Expression of CD11b (ITGAM) and CD86 in different French-American-British subtypes of AML from TCGA (left panels) and TARGET (right panels) datasets. ${ }^{* *}$ P $<0.01$ and ${ }^{* * *}$ P $<0.001$. (B) Different AML cell lines (except for KG1a) analysed using FCM (presented in histogram) revealed that chidamide causes upregulation of the levels of the surface marker CD11b. The mean fluorescence intensity is also presented on the right. (C) Four primary AML samples analysed using FCM (as presented in the histogram) revealed that chidamide $(2.5 \mu \mathrm{M})$ caused upregulation of CD86. P1, patient 1; AML, acute myeloid leukaemia; TCGA, The Cancer Genome Atlas; APC, allophycocyanin; PE, phycoerythrin; CY7, cyanin 7; FCM, flow cytometry; ITGAM, integrin subunit $\alpha$ M.
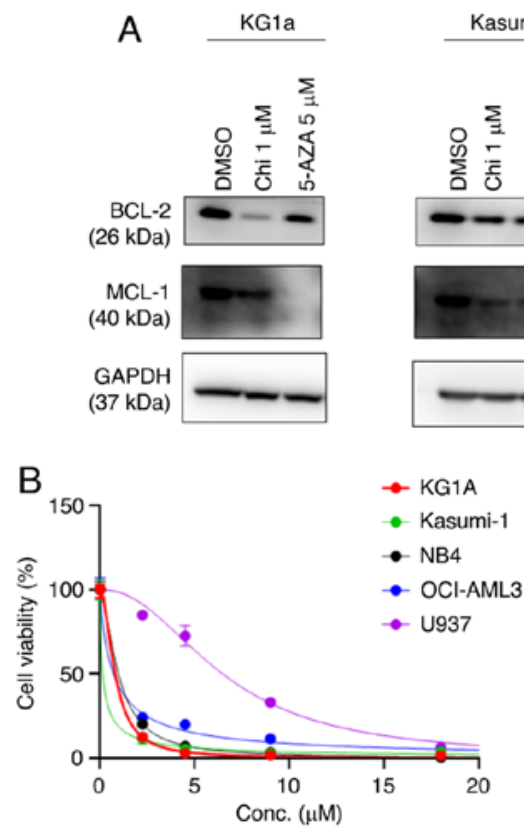

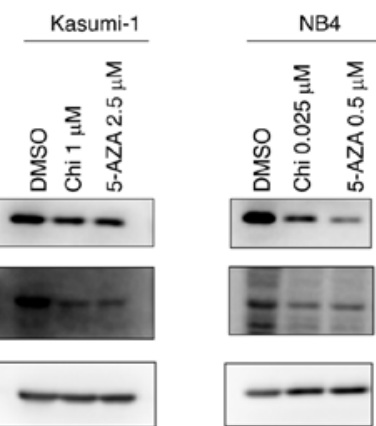

C

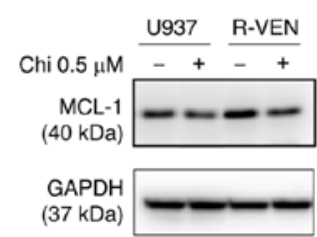

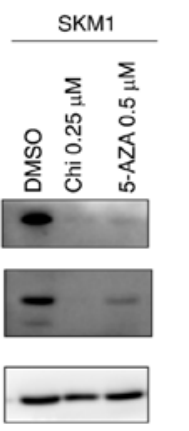

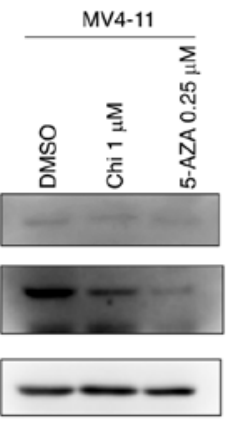

D

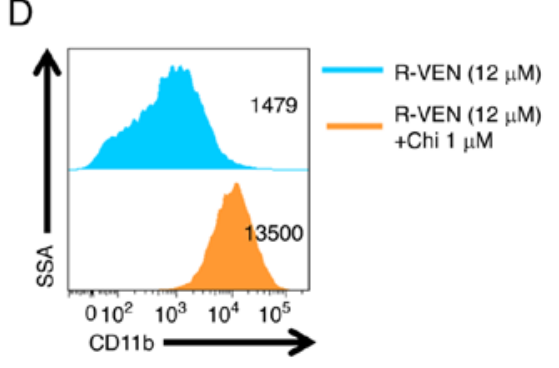

Figure 5. Effect of chidamide and 5-AZA treatment alone on the protein expression levels of BCL-2 and MCL-1 in the different AML cell lines and venetoclax-resistant U937 cell line. (A) All of the cell lines were treated with suitable concentrations of the two drugs for $72 \mathrm{~h}$ and GAPDH was used as an internal control. (B) Venetoclax inhibited cell proliferation in different AML cell lines (M1-M5). All of the AML cell lines were exposed to venetoclax for $72 \mathrm{~h}$ and cell viability was subsequently determined using a Cell Counting Kit- 8 assay. (C) MCL-1 protein levels were detected by western blot analysis after 24 h of chidamide treatment. (D) Treatment with $1 \mu \mathrm{M}$ chidamide in R-VEN (12 $\mu \mathrm{M})$ for $72 \mathrm{~h}$ caused a more significant increase in the expression of the myeloid differentiation marker CD11b. Chi, chidamide; R-VEN (12 $\mu \mathrm{M})$, U937 cell line resistant to $12 \mu \mathrm{M}$ venetoclax; 5-AZA, 5-azacitidine; AML, acute myeloid leukaemia; MCL-1, myeloid-cell leukemia 1. 
BCL-2 family proteins are important for cell fate, as they may regulate cells in two ways: Anti- and pro-apoptosis $(33,34)$. Our previous research also found that chidamide is able to induce apoptosis in AML cell lines via antiapoptotic BCL-2 family proteins (BCL-2 and MCL-1) (35). However, in the present study, no significant synergistic effect of chidamide combined with 5-AZA to downregulate the BCL-2 and MCL-1 was observed (data not shown). In addition, chidamide was also able to downregulate the expression of BCL-XL and upregulate BAX in MV4-11 and Kasumi-1. Various HDACIs (in combination with decitabine) shortened tumour cell survival at the mRNA and protein levels (36). The results of the present study demonstrated that chidamide and 5-AZA alone were able to degrade the antiapoptotic proteins BCL-2 and MCL-1 in several AML cell lines. Of note, it was observed that the expression of MCL-1 was compensatorily increased in the venetoclax-resistant U937 cell line (35). Chidamide degraded MCL-1 in venetoclax-resistant U937 cells and upregulated the expression of CD11b, and venetoclax slightly promoted CD11b expression in venetoclax-resistant U937 cells as compared with U937 (data not shown). There is reason to believe that the downregulation of MCL-1 may be a promising strategy for the treatment of venetoclax-resistant leukaemia. Further investigations will be performed in in vivo models (e.g. PDX animal models or an increased number of patient samples).

Other studies have reported that chidamide is able to degrade the AML1-ETO fusion gene and also inhibit leukaemia cells with mixed lineage leukemia (MLL) rearrangements $(37,38)$. Chidamide may become a novel option in the treatment of certain subtypes of AML, such as $t(8 ; 21)$ and MLL rearrangement, in the future.

It has been demonstrated that antitumour drugs have multiple functions and targets. HDACI combined with 5-AZA may have multiple pathways for inducing cell apoptosis, differentiation and proliferation inhibition. In conclusion, chidamide in combination with 5-AZA has a synergistic antileukaemia effect in vitro and may be considered a therapeutic strategy for AML.

\section{Acknowledgements}

Not applicable.

\section{Funding}

This work was supported by grants from the Social Development-Clinical Frontier Project of Jiangsu Province (grant no. BE2018652), the National Natural Science Foundation of China ( grant no. 81970138), Translational Research Grant of the National Clinical Research Center for Hematology (grant no. 2020ZKMB05) and the Project of State Key Laboratory of Radiation Medicine and Protection (grant no. GZK1202002).

\section{Availability of data and materials}

The datasets used and/or analyzed during the current study are available from the corresponding author on reasonable request.

\section{Authors' contributions}

HYQ contributed conceptualization, funding acquisition, supervision, resources, study design and confirmed the authenticity of all the raw data. SLX contributed to conceptualization, funding acquisition, supervision, resources, study design and confirmed the authenticity of all the raw data. ZL designed and performed the experiments. JZ, MZ and JLL performed the experiments. QCQ and JHF analyzed data. ZL wrote the manuscript. All authors read and approved the final manuscript.

\section{Ethics approval and consent to participate}

The present study was approved by The First Affiliated Hospital of Soochow University (Suzhou, China) and all patients provided written informed consent.

\section{Patient consent for publication}

Not applicable.

\section{Competing interests}

The authors declare that they have no competing interests.

\section{References}

1. Dawson MA and Kouzarides T: Cancer epigenetics: From mechanism to therapy. Cell 150: 12-27, 2012.

2. Bhatla T, Wang J, Morrison DJ, Raetz EA, Burke MJ, Brown P and Carroll WL: Epigenetic reprogramming reverses the relapse-specific gene expression signature and restores chemosensitivity in childhood B-lymphoblastic leukemia. Blood 119: 5201-5210, 2012.

3. Baylin SB and Jones PA: A decade of exploring the cancer epigenome-biological and translational implications. Nat Rev Cancer 11: 726-734, 2011.

4. Roy DM, Walsh LA and Chan TA: Driver mutations of cancer epigenomes. Protein Cell 5: 265-296, 2014.

5. Sun Y, Chen BR and Deshpande A: Epigenetic regulators in the development, maintenance, and therapeutic targeting of acute myeloid leukemia. Front Oncol 8: 41, 2018.

6. Figueroa ME, Lugthart S, Li Y, Erpelinck-Verschueren C, Deng X, Christos PJ, Schifano E, Booth J, van Putten W, Skrabanek L, et al: DNA methylation signatures identify biologically distinct subtypes in acute myeloid leukemia. Cancer Cell 17: 13-27, 2010.

7. Jiang Y, Dunbar A, Gondek LP, Mohan S, Rataul M, O'Keefe C, Sekeres M, Saunthararajah Y and Maciejewski JP: Aberrant DNA methylation is a dominant mechanism in MDS progression to AML. Blood 113: 1315-1325, 2009.

8. Fey MF, Buske C; ESMO Guidelines Working Group: Acute myeloblastic leukaemias in adult patients: ESMO clinical practice guidelines for diagnosis, treatment and follow-up. Ann Oncol 24 (Suppl 6): vi138-vi143, 2013.

9. Fenaux P, Haase D, Santini V, Sanz GF, Platzbecker U and Mey U; ESMO Guidelines Committee. Electronic address: Clinicalguidelines@esmo.org: Myelodysplastic syndromes: ESMO clinical practice guidelines for diagnosis, treatment and follow-up ${ }^{\text {th }}$. Ann Oncol 32: 142-156, 2021.

10. Seto E and Yoshida M: Erasers of histone acetylation: The histone deacetylase enzymes. Cold Spring Harb Perspect Biol 6: a018713, 2014.

11. Tan P, Wei A, Mithraprabhu S, Cummings N, Liu HB, Perugini M, Reed K, Avery S, Patil S, Walker P, et al: Dual epigenetic targeting with panobinostat and azacitidine in acute myeloid leukemia and high-risk myelodysplastic syndrome. Blood Cancer J 4: e170, 2014.

12. Viré E, Brenner C, Deplus R, Blanchon L, Fraga M, Didelot C, Morey L, Van Eynde A, Bernard D, Vanderwinden JM, et al: The polycomb group protein EZH2 directly controls DNA methylation. Nature 439: 871-874, 2006 
13. Gong K, Xie J, Yi H and Li W: CS055 (Chidamide/HBI-8000), a novel histone deacetylase inhibitor, induces G1 arrest, ROS-dependent apoptosis and differentiation in human leukaemia cells. Biochem J 443: 735-746, 2012

14. Xu Y, Zhang P and Liu Y: Chidamide tablets: HDAC inhibition to treat lymphoma. Drugs Today (Barc) 53: 167-176, 2017.

15. Xu F, Guo H, Shi M, Liu S, Wei M, Sun K and Chen Y: A combination of low-dose decitabine and chidamide resulted in synergistic effects on the proliferation and apoptosis of human myeloid leukemia cell lines. Am J Transl Res 11: 7644-7655, 2019.

16. Jaatinen $\mathrm{T}$ and Laine $\mathrm{J}$ : Isolation of mononuclear cells from human cord blood by ficoll-paque density gradient. Curr Protoc Stem Cell Biol Chapte: Jun 1, 2007 (Epub ahead of print). doi: 10.1002/9780470151808.sc02a01s1.

17. Ambavaram MM and Pereira A: Setting up reverse transcription quantitative-PCR experiments. Methods Mol Biol 678: 45-54, 2011.

18. Pollyea DA: Therapeutic advances in first-line management of acute myeloid leukemia. J Natl Compr Canc Netw 17: 1441-1443, 2019.

19. Döhner H, Estey EH, Amadori S, Appelbaum FR, Büchner T, Burnett AK, Dombret H, Fenaux P, Grimwade D, Larson RA, et al Diagnosis and management of acute myeloid leukemia in adults: Recommendations from an international expert panel, on behalf of the European LeukemiaNet. Blood 115: 453-474, 2010.

20. Ganesan A, Nolan L, Crabb SJ and Packham G: Epigenetic therapy: Histone acetylation, DNA methylation and anti-cancer drug discovery. Curr Cancer Drug Targets 9: 963-981, 2009.

21. Anzai H, Frost P and Abbruzzese JL: Synergistic cytotoxicity with 2'-deoxy-5-azacytidine and topotecan in vitro and in vivo. Cancer Res 52: 2180-2185, 1992.

22. Garcia-Manero G, Kantarjian HM, Sanchez-Gonzalez B, Yang H, Rosner G, Verstovsek S, Rytting M, Wierda WG, Ravandi F, Koller C, et al: Phase 1/2 study of the combination of 5-aza-2'-deoxycytidine with valproic acid in patients with leukemia. Blood 108: 3271-3279, 2006.

23. Najem SA, Khawaja G, Hodroj MH, Babikian P and Rizk S: Adjuvant epigenetic therapy of decitabine and suberoylanilide hydroxamic acid exerts anti-neoplastic effects in acute myeloid leukemia cells. Cells 8: 1480, 2019.

24. Bewersdorf JP, Shallis R, Stahl M and Zeidan AM: Epigenetic therapy combinations in acute myeloid leukemia: What are the options? Ther Adv Hematol 10: 2040620718816698, 2019.

25. Lübbert $M$ and Kuendgen A: Combining DNA methyltransferase and histone deacetylase inhibition to treat acute myeloid leukemia/myelodysplastic syndrome: Achievements and challenges. Cancer 121: 498-501, 2015.

26. Liu Z, Ding K, Li L, Liu H, Wang Y, Liu C and Fu R: A novel histone deacetylase inhibitor Chidamide induces G0/G1 arrest and apoptosis in myelodysplastic syndromes. Biomed Pharmacother 83: 1032-1037, 2016.

27. Sandor V, Senderowicz A, Mertins S, Sackett D, Sausville E, Blagosklonny MV and Bates SE: P21-dependent g(1)arrest with downregulation of cyclin D1 and upregulation of cyclin $\mathrm{E}$ by the histone deacetylase inhibitor FR901228. Br J Cancer 83: 817-825, 2000 .
28. Vijayaraghavalu S, Dermawan JK, Cheriyath V and Labhasetwar V: Highly synergistic effect of sequential treatment with epigenetic and anticancer drugs to overcome drug resistance in breast cancer cells is mediated via activation of p21 gene expression leading to G2/M cycle arrest. Mol Pharm 10: 337-352, 2013.

29. Hassan K, Bukhari KP, Zafar A, Malik MZ and Akhtar MJ: Acute leukaemia in children-French-American-British (FAB) classification and its relation to clinical features. J Pak Med Assoc 42: 29-31, 1992

30. Heo SK, Noh EK, Yoon DJ, Jo JC, Koh S, Baek JH, Park JH, Min YJ and Kim H: Rosmarinic acid potentiates ATRA-induced macrophage differentiation in acute promyelocytic leukemia NB4 cells. Eur J Pharmacol 747: 36-44, 2015.

31. Sykes DB, Kfoury YS, Mercier FE, Wawer MJ, Law JM, Haynes MK, Lewis TA, Schajnovitz A, Jain E, Lee D, et al: Inhibition of dihydroorotate dehydrogenase overcomes differentiation blockade in acute myeloid leukemia. Cell 167: 171-186 e15, 2016.

32. Re F, Arpinati M, Testoni N, Ricci P, Terragna C, Preda P, Ruggeri D, Senese B, Chirumbolo G, Martelli V, et al: Expression of CD86 in acute myelogenous leukemia is a marker of dendritic/monocytic lineage. Exp Hematol 30: 126-134, 2002.

33. Krishna S, Low IC and Pervaiz S: Regulation of mitochondrial metabolism: Yet another facet in the biology of the oncoprotein Bcl-2. Biochem J 435: 545-551, 2011.

34. Ebrahim AS, Sabbagh H, Liddane A, Raufi A, Kandouz M and Al-Katib A: Hematologic malignancies: Newer strategies to counter the BCL-2 protein. J Cancer Res Clin Oncol 142: 2013-2022, 2016

35. Wang BR, Wan CL, Liu SB, Qiu QC, Wu TM, Wang J, Li YY, Ge SS, Qiu Y, Shen XD, et al: A combined histone deacetylases targeting strategy to overcome venetoclax plus azacitidine regimen resistance in acute myeloid leukaemia: Three case reports. Front Oncol 11: 797941, 2021.

36. Kaminskyy VO, Surova OV, Vaculova A and Zhivotovsky B: Combined inhibition of DNA methyltransferase and histone deacetylase restores caspase-8 expression and sensitizes SCLC cells to TRAIL. Carcinogenesis 32: 1450-1458, 2011.

37. Al- Harbi S, Aljurf M, Mohty M, Almohareb F and Ahmed SOA: An update on the molecular pathogenesis and potential therapeutic targeting of AML with $\mathrm{t}(8 ; 21)(\mathrm{q} 22 ; \mathrm{q} 22.1) ; \mathrm{RUNX} 1-\mathrm{RUNX} 1 \mathrm{~T} 1$. Blood Adv 4: 229-238, 2020.

38. Ahmad K, Katryniok C, Scholz B, Merkens J, Löscher D, Marschalek R and Steinhilber D: Inhibition of class I HDACs abrogates the dominant effect of MLL-AF4 by activation of wild-type MLL. Oncogenesis 3: e127, 2014.

This work is licensed under a Creative Commons Attribution-NonCommercial-NoDerivatives 4.0 International (CC BY-NC-ND 4.0) License. 